\title{
Study of the first productive active region in solar cycle 24
}

\author{
L. P. Li, J. Zhang, T. Li, S. H. Yang, and Y. Z. Zhang
}

\begin{abstract}
Key Laboratory of Solar Activity, National Astronomical Observatories, Chinese Academy of Sciences, 100012 Beijing, PR China e-mail: lepingli@nao.cas.cn
\end{abstract}

Received 21 September 2010 / Accepted 9 December 2011

\begin{abstract}
Context. The Sun is very quiet with less sunspots and activity since the beginning of solar cycle 24 . However, the active region (AR) 11045 emerged on February 5, 2010, is associated with 43 (8 M- and $35 \mathrm{C}$-class) flares, 53 coronal mass ejections (CMEs), 29 filament eruptions, 19 extreme ultraviolet (EUV) waves and abundant jets, indicating that this AR is the first productive one of solar cycle 24 .

Aims. We study the AR evolution and its associated activities, and also their relationships, to understand this productive AR.

Methods. We used SOHO/MDI magnetograms to study the magnetic fields, STEREO/SECCHI images to explore the activities, and GOES measurements to investigate the soft X-ray flux of the AR.

Results. During the AR evolution, six pairs of main magnetic fields emerged, and $93.1 \%$ flares and $82.75 \%$ filament eruption occurred in the emergence and stable phases of the magnetic flux. However, 43.4\% CMEs occurred in the decaying phase, even though there were less flares. An example is given to show that an event is related to a flare, a filament eruption, a CME and an EUV wave from inner corona to outer corona in space, and the filament eruption and EUV wave occur near the peak time of the flare. Among the 29 filament eruptions, $79.3 \%$ are associated with CMEs, as well as 58.6\%, associated with flares, and 34.5\%, associated with EUV waves. During the 12-day active phase, 575 jets are detected with a daily occurrence rate of 49.3. This is the first time that so many jets have been identified in one AR, implying at least 575 lower magnetic reconnection processes during the AR evolution. We statistically studied these jets along with the AR evolution, and noticed that the jets mostly occurred surrounding the emerging flux. We also investigated the spatio-temporal relationships between the jets and the flares, and find that the jets are usually rooted around the flare cores, and the soft X-ray flux is inverse correlated with the number of the jets, especially during the beginning 9 days since the AR emergence. In comparison with AR 11045, we studied the other newly emerging AR 11045, and obtained similar results. The relationships between the jets and the flares may well represent a scenario of two-step magnetic reconnection. Using schematic diagrams, we explain the remarkable magnetic field emergence, cancelation and shear motion of AR 11045, and its associated activities.
\end{abstract}

Key words. Sun: flares - Sun: filaments, prominences - magnetic fields - Sun: activity - Sun: coronal mass ejections (CMEs) sunspots

\section{Introduction}

Solar active regions (ARs), displayed as plages and sunspots, are concentrations of intense magnetic fields in the solar atmosphere. They are composed of flux tubes, which are thought to be formed near the tachocline of the Sun (Miesch 2005). The turbulent sub-surface forces result in a buoyant upward motion that causes the formation of $\Omega$-type loops (Magara \& Longcope 2001; Fan 2001) or serpentine field lines (Magara 2001; Pariat et al. 2004, 2007), which in turn emerge through the photosphere to form ARs (Conlon et al. 2010). Sunspots are the visible footpoints of these AR loops, and appear as collections of positive and negative polarity magnetic flux elements in magnetograms. These highly dynamic, complicated loops are rooted in the photosphere (Patterson \& Zirin 1981; Qiu \& Gary 2003), extend into the corona, and are the source of many extreme solar events, such as flares (Schmieder et al. 1996; Wang et al. 2000; Zhang et al. 2001a; Isobe et al. 2002; Schrijver et al. 2009), coronal mass ejections (CMEs) (Harrison 1986; Sheeley et al. 1999; Webb et al. 2000; Zhang et al. 2001a, b), filament eruptions (Sterling \& Moore 2005; Tripathi et al. 2006; Liu \& Wang 2009), waves (Aschwanden et al. 1999; Nakariakov \& Ofman 2001; Wang et al. 2003), surges (Roy 1973a; Canfield et al. 1996; Jiang et al. 2007) and jets (Canfield et al. 1996; Cook et al. 1996; Chae et al. 1999; Zhang et al. 2000; Pariat et al. 2009a; Archontis et al. 2010). The formation and evolution of ARs are therefore continuously attracting the attention of many observers and theorists (Martin \& Harvey 1979; Golub et al. 1981; Lites et al. 1998; Gibson et al. 2002).

Transient, small-scale ejections of plasma from the lower atmosphere are common manifestations of solar activity. Cool plasma ejections were observed as emission in $\mathrm{H} \alpha$ or absorption at other wavelengths and historically called surges (Newton 1934). Surges have been studied for more than 50 years (see Roy 1973a, b). They are straight or slightly curved mass ejections stretching out and away from small flare-like brightenings at footpoints in the chromosphere into coronal heights, which move upward at $20-200 \mathrm{~km} \mathrm{~s}^{-1}$, reach heights of up to $200000 \mathrm{~km}$, and typically last for 10-20 min (Roy 1973a). During the descending stage, the matter in a surge falls down along almost the same path that it took in the ascending phase. Surges usually show a strong trend to recurrence (Jiang et al. 2007), such that they appear at the same position many times with a duration of 10-20 min and a repetition rate of an hour (Tandberg-Hanssen 1977; Schmieder et al. 1984). The rotational or helical motions were, on occasions, also observed in surge activity (Canfield et al. 1996; Jibben \& Canfield 2004).

Similar hot ejection phenomena were usually called jets, and observed as emission in ultraviolet (UV; Brueckner \& Bartoe 1983), extreme ultraviolet (EUV; Schmahl 1981; Alexander \& Fletcher 1999) and soft X-ray (Shibata et al. 1992). Very 
early observations of jets were provided by Skylab, and later by Yohkoh in soft X-ray (Tsuneta et al. 1991; Shimojo et al. 1996). X-ray jets are linear structures with an average length of $2 \times 10^{5} \mathrm{~km}$, average peak velocity of $190 \mathrm{~km} \mathrm{~s}^{-1}$ and lifetimes ranging from 100 to $16000 \mathrm{~s}$, and they are always associated with small flares (Shibata et al. 1992; Shimojo et al. 1996). Moreover, Chae et al. (1999) reported that the TRACE EUV jets are very similar to the Yohkoh X-ray jets in shape, velocity, and in the characteristic of their association with microflares, except for the fact that the EUV jets are much smaller $\left(0.4-1 \times 10^{4} \mathrm{~km}\right)$ and shorter lived (a few minutes) than X-ray jets. In many cases, the appearance of jets is also recurrent. Chifor et al. (2008) have indicated a recurrent jet emission in an AR, and Wang \& Sheeley (2002) found numerous jet-like ejections originating from ARs located inside or near the boundaries of non-polar coronal holes.

Some observations have shown that cool surges and hot jets are closely related in space and time (Rust et al. 1977; Schmieder et al. 1994; Alexander \& Fletcher 1999). Schmieder et al. (1994) found a delay of 3-4 min between cool and hot plasma emission. Alexander \& Fletcher (1999) showed that the maximum extent time of an X-ray jet precede that of in EUV by $5 \mathrm{~min}$, and they explained this delay as being caused by the cooling of the earlier, hotter jet material. Jiang et al. (2007) also reported that the cool components always occurred later than the hot components. On the other hand, Rust et al. (1977) found that few $\mathrm{H} \alpha$ surges were coincident with noticeable changes in X-ray emission. Schmieder et al. (1988) compared $\mathrm{H} \alpha$ surges with UV and $\mathrm{X}$-ray data, and supported the idea that $\mathrm{H} \alpha$ surges are spatially correlated with UV and X-ray emission. Shibata et al. (1992) presented an example of an X-ray jet that is nearly co-spatial with an $\mathrm{H} \alpha$ surge, and Schmieder et al. (1994) also found a jetlike X-ray emission thread that overlies the brightest thread of $\mathrm{H} \alpha$ emission in $\mathrm{H} \alpha$ surges. However, Canfield et al. (1996) reported that X-ray jets are not co-spatial with $\mathrm{H} \alpha$ surges even though they are associated with each other. Similar results were also obtained by Chae et al. (1999) and Jiang et al. (2007).

Jet-like activities are often associated with certain types of magnetic activity around their bases in the photosphere, such as satellite polarity (Rust 1968), evolving magnetic features (Roy 1973a), and moving magnetic bipoles (Canfield et al. 1996). Kurokawa \& Kawai (1993) noticed that $\mathrm{H} \alpha$ surges were often observed at the very early stage in some emerging flux regions and recurred for many hours. Shimojo et al. (1998) found that $\mathrm{X}$-ray jets also favored the regions of evolving magnetic flux (increasing or decreasing) in their statistical study. Chae et al. (1999), Zhang et al. (2000), Liu \& Kurokawa (2004) and Jiang et al. (2007) showed that surges are closely correlated with magnetic flux cancelations at their footpoints, forced by the newly emerging flux with a preexisting ambient field. In theory, many models were devoted to the interpretation of solar dynamic phenomena such as surges and jets (see the detailed discussion by Schmieder et al. 1995; Canfield et al. 1996). It is well accepted that surges are closely correlated with newly emerging flux in the periphery of sunspots or high-flux concentrations. They are manifestations of the magnetic reconnection between newly emerging and pre-existing flux. Yokoyama \& Shibata (1996) successfully showed that not only hot plasma can be accelerated by magnetic reconnection driven by emerging flux using numerical simulations but cool plasma as well.

According to "The Weekly" reported by NOAA/Space Weather Prediction Center, the first sunspot of the solar cycle (SC) 24 was observed on January 4, 2008, and it was numbered NOAA AR 10981. Later, NOAA ARs 10990 and 10993 were observed in April and May, 2008, respectively, and were also classified as sunspots of SC 24. On the other hand, sunspots belonging to SC 23 had also been appearing near the equator until and even after mid 2008 (Bhatt et al. 2009). Therefore, the year 2008 is considered as the transition period from SC 23 to SC 24. As expected by some authors, SC 24 will be somewhat weaker compared with SCs 21-23 (Jiang \& Wang 2007; Li 2009). Indeed, the Sun was staying at a fairly low activity level with less sunspots and activities until February 2010, belonging to the new cycle since its beginning. However, on February 5, 2010, NOAA AR 11045, belonging to SC 24, emerged on the solar disk at heliographic position N23 E32. Along with the complicated evolution of the AR, a mass of M-class flares, CMEs, filament eruptions, EUV waves, and abundant jets associated with the AR occurred, implying that this $\mathrm{AR}$ is the first productive one of SC 24.

In this paper, we study the formation and evolution of AR 11045, the first productive AR in SC 24, and its associated solar activities, such as flares, CMEs, filament eruptions, EUV waves and jets. The data and observations are described in Sect. 2. We present the results in Sect. 3 and the conclusions and a brief discussion in Sect. 4.

\section{Data and observations}

We employed the magnetograms of the Solar and Heliospheric Observatory Michelson Doppler Imager (SOHO/MDI; Scherrer et al. 1995) to investigate the formation and evolution of the magnetic fields of AR 11045. The time cadence and pixel size of the MDI magnetograms are $96 \mathrm{~min}$ and $1.96^{\prime \prime}$, respectively. We used the measurements of the SOHO Extreme ultraviolet Imaging Telescope (SOHO/EIT; Delaboudinière et al. 1995) and the Solar TErrestrial RElation Observatory (STEREO; Howard et al. 2008) Extreme UltraViolet Imager (EUVI) to study the solar activities, including flares, filament eruptions, EUV waves and jets. The time cadences of the SOHO/EIT $195 \AA$, STEREO/EUVI $195 \AA$ and $304 \AA$ images are 12,5 and $10 \mathrm{~min}$, separately, as well as the pixel sizes, 2.63", 1.59" and 1.59", respectively. We also employed the STEREO/COR1, COR2 and SOHO Large Angle and Spectrometric Coronagraph (SOHO/LASCO; Bruechner et al. 1995) images to research CMEs, and the Geostationary Operational Environmental Satellite (GOES; Thomas et al. 1995) data, to explore the soft X-ray fluxes associated with the AR.

The SOHO observations of AR 11045 were made from February 5 to 14 , because the AR rotated out of sight after February 14. However, because of the special positions of the STEREO A and B satellites, there are 15 day (from February 5 to 19) STEREO observations of the AR. Figure 1 illustrates the positions of the STEREO A and B satellites related to the Sun and the Earth at 2:00 UT on February 7, 2010. From February 5 to 19, 2010, the separation angles between STEREO$\mathrm{A}$ and SOHO, SOHO and STEREO-B, and STEREO-A and STEREO-B, are in the ranges of $64.8^{\circ}-65.3^{\circ}, 70.7^{\circ}-71.2^{\circ}$, and $135.5^{\circ}-136.5^{\circ}$, respectively. The long-duration observations provide a good opportunity to study the AR dynamics and its associated solar activities.

\subsection{Magnetic field evolution of $A R 11045$}

Figure 2 displays series of SOHO/MDI magnetograms, illustrating the formation and evolution of the magnetic fields of AR 11045, among which the white patches represent the positive 


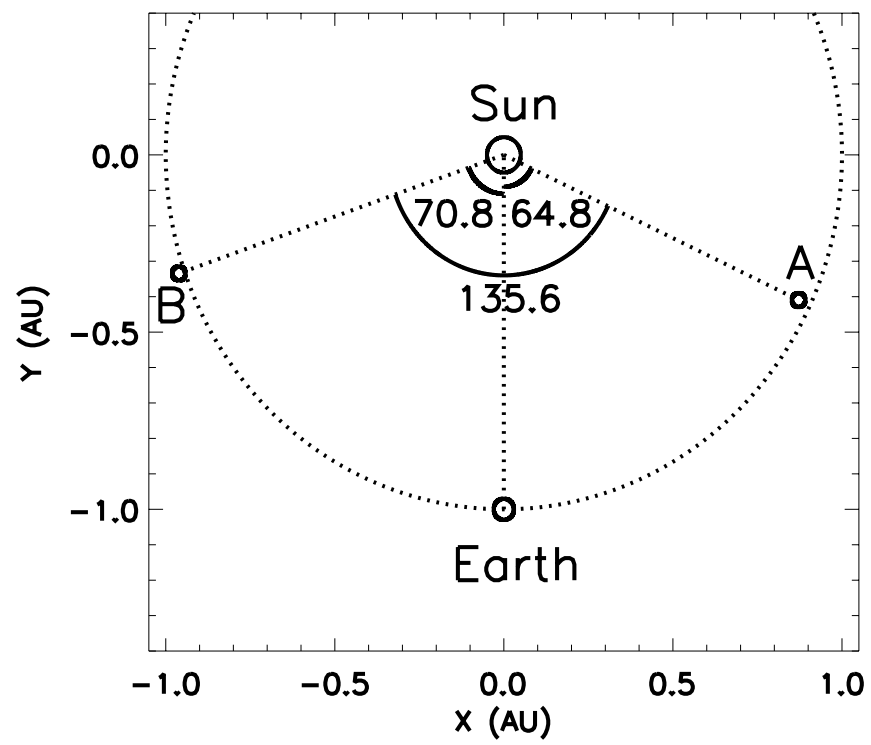

Fig. 1. Diagram displaying the positions of the Sun, the Earth and the STEREO A and B satellites at 2:00 UT on February 7, 2010.

magnetic fields, and the black ones, the negative magnetic fields. To better show the formation and evolution of the AR magnetic fields, we display a movie associated with Fig. 2, which shows the time sequence of SOHO/MDI line of sight magnetograms with a cadence of $96 \mathrm{~min}$. Despite the presence of several pairs of minor emerged magnetic fields, we discovered six pairs of main magnetic field emergences during the evolution of the AR. The first pair of magnetic fields, marked as $A$ and $B$ in Fig. 2a, appeared at about 8:03 UT on February 5, 2010, and then separated. After several hours, the second pair of magnetic fields, depicted as $C$ and $D$ in Fig. 2b, emerged to the west of the negative magnetic field $B$, and showed separation motion immediately after the emergence. Subsequently, the cancelation between the negative magnetic field $B$ and the positive one $C$ occurred, resulting in the disappearance of these two magnetic fields. Followed by the second pair of magnetic field emergence, the third pair of magnetic fields, denoted by $E$ and $F$ in Fig. 2c, appeared to the southwest of the negative magnetic field $D$ from about 19:15 UT, and moved apart. Afterward, the cancelation and shear motion between the magnetic fields $D$ and $E$ took place. At the same time, the fourth pair of magnetic fields $G$ and $H$ (see Fig. 2c) emerged to the north of the magnetic fields $C$ and $D$. From 8:03 UT on February 7, 2010, the fifth pair of magnetic fields, indicated by $I$ and $J$ in Fig. 2d, emerged to the south of the magnetic fields $A$ and $G$, accompanied by obvious shear motion and cancelation between the magnetic fields $G$ and $J$, as well as $E$ and $J$. Thereafter, the positive magnetic field $E$ disappeared. From 17:39 UT on February 8, 2010, the sixth pair of magnetic fields $L$ and $K$, displayed in Fig. 2f, emerged to the south of the magnetic field $J$, and separated. Finally, as a result of the separation, cancelation and shear motion of the emerged magnetic fields, AR 11045 became less complicated (see Fig. 2f), looking like a big and simple magnetic bipole.

We measured the magnetic fluxes of the AR, and display them in Fig. 3. In this figure, the black curve represents the total unsigned magnetic flux, the blue one the positive magnetic flux, the red and the yellow ones the absolute values of the negative magnetic flux and the net magnetic flux. It indicates that the total magnetic flux began to rise from about the middle of February 5, and achieved the maximum flux, $2.1 \times 10^{22} \mathrm{Mx}$, in the middle of February 8, with an emergence rate of $2.9 \times 10^{20} \mathrm{Mx} \mathrm{h}^{-1}$.
Meanwhile, the net magnetic flux increased from zero to the maximum value of $3.1 \times 10^{21} \mathrm{Mx}$. Later the magnetic flux gradually decreased. According to the magnetic flux, we distinguish three different phases in the evolution of the AR, including the emergence phase, from 8:00 UT on February 5 to 14:00 UT on February 8, the stable phase, from 14:00 UT on February 8 to 15:00 UT on February 10, and the decaying phase, from 15:00 UT on February 10 to the end of the AR. The red vertical dashed lines in Fig. 3 represent the two times, 14:00 UT on 8 February and 15:00 UT on 10 February, which separate the three different phases of the AR.

\section{Results}

\subsection{Flares, CMEs, filament eruptions, and EUV waves in $A R 11045$}

Using the SOHO/EIT observations from February 5 to 14, we find that most of the magnetic field evolutions, such as emergences, cancelations and shear motions, are associated with EUV brightenings or other solar activities. Employing the continual STEREO/EUVI measurements, we investigated these activities, and note that there are many solar activities associated with the AR from February 5 to 16, but few solar activity after February 16 when the AR became bright patches. This indicates that the 12-day interval from February 5 to 16 is the active phase of the AR, and the AR nearly disappeared after February 16. From the GOES soft X-ray fluxes, we identified 43 (35 C-class and $8 \mathrm{M}$-class) flares ${ }^{1}$ during the active phase of the AR. Based on COR1 preliminary CME list $^{2}$ and COR 1 daily movies ${ }^{3}$, we identified $53 \mathrm{CMEs}^{1}$ associated with the AR, in conjunction with the EUVI observations. Using the STEREO/EUVI $304 \AA^{\circ}$ movies $^{1}$ and $195 \AA$ movies of AR 11045 , we found 29 filament eruptions ${ }^{1}$, and 19 EUV waves ${ }^{1}$ during the AR active phase. Table 1 lists the information of $8 \mathrm{M}$-class flares and their associated 5 CMEs and 3 filament eruptions, of which the event on February 12 has been studied by Kumar et al. (2011). Columns 1-6 in Table 1 show the date, the beginning time, the peak time, the ending time, the class and the heliographic position of the flares, and Cols. 7 and 8 show whether there are CMEs associated with the flares and the initial time of the CMEs appeared in STEREO/COR1, respectively. Column 9 indicates whether there are filament eruptions associated with the flares. It indicates that $5(62.5 \%)$ M-class flares are associated with CMEs.

Figure 4 displays an example of the 8 M-class flares on February 7, 2010, when the separation angles between the STEREO-A and the SOHO, the SOHO and the STEREO-B, and the STEREO-A and the STEREO-B are $64.8^{\circ}, 70.8^{\circ}$ and $135.6^{\circ}$, respectively (see Fig. 1). The flare event is an M 6.4 one from 2:20 to 2:39 UT with a peak time of 2:34 UT. It is associated with a filament eruption, a CME and an EUV wave. Figures 4a, b and i are EUVI-A $304 \AA$, EUVI-B $304 \AA$ and EIT $195 \AA$ images showing a flare and an erupting filament. Figures $4 c, d$ and $j$ are EUVI-A, EUVI-B and EIT 195 A running ratio images illustrating an EUV wave associated with the flare and the filament eruption. These display that the EUV wave is a dome-shaped large-scale coronal wave with a filament eruption behind/inside the dome. Figures $4 \mathrm{e}-\mathrm{h}$ illustrate a $\mathrm{CME}$ associated with the flare observed by COR1-A, COR1-B, COR2-A and COR2-B,

\footnotetext{
${ }^{1}$ http://ourstar.bao.ac.cn/lepingli/AR11045.htm

2 http://cor1.gsfc.nasa.gov/catalog/cme/2010/

${ }^{3}$ http://cor1.gsfc.nasa.gov/dailymov/dailymov/GIF/
} 


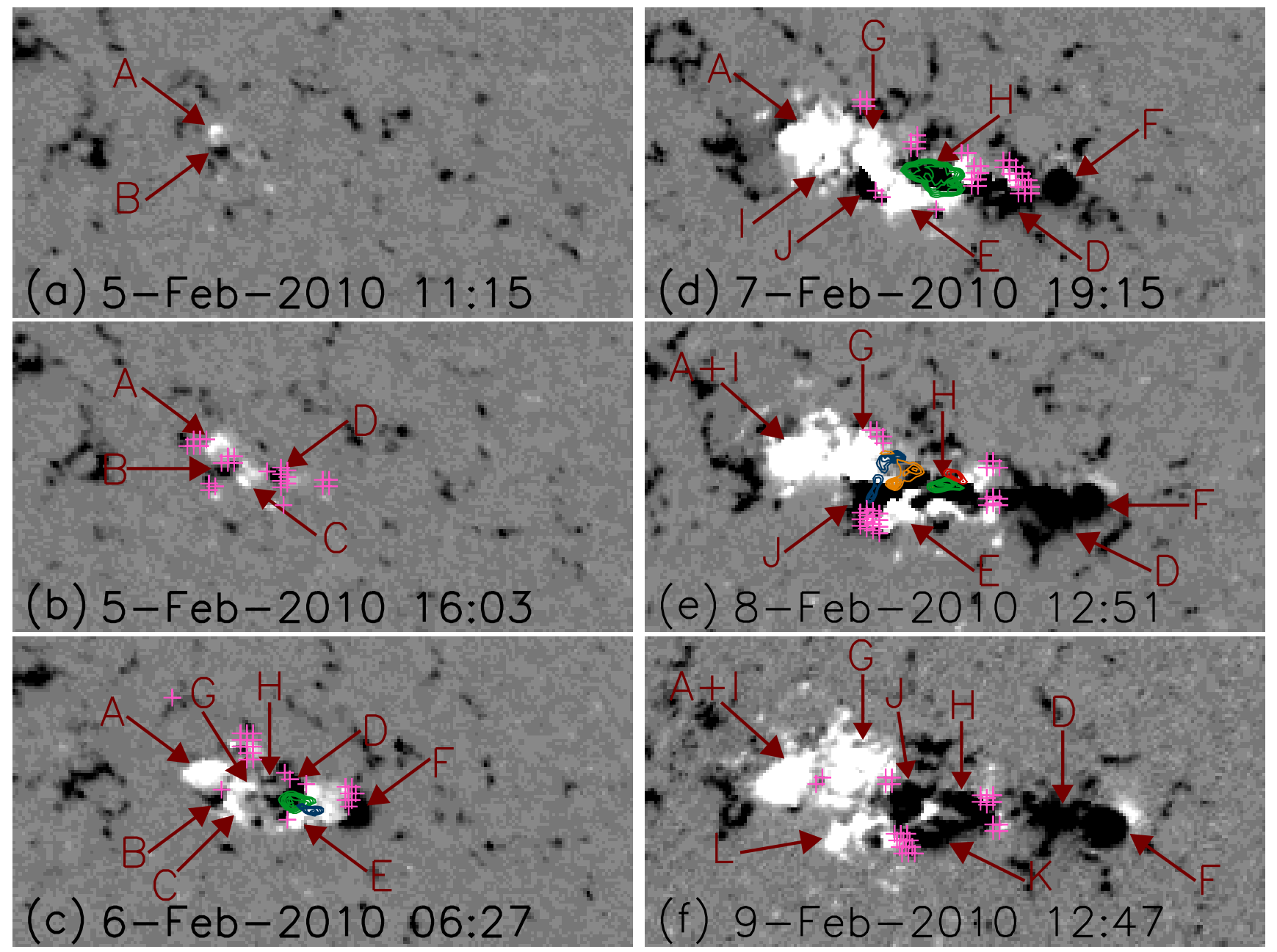

Fig. 2. Time series of MDI magnetograms illustrating the formation and evolution of the magnetic fields of the active region (AR) 11045 . Arrows A-L mark the emerged magnetic fields. The pink pluses represent the roots of the jets, and the colored contours seven M-class flare cores. The field of view (FOV) is $400^{\prime \prime} \times 200^{\prime \prime}$. (An animation of this figure is available in the online journal.)

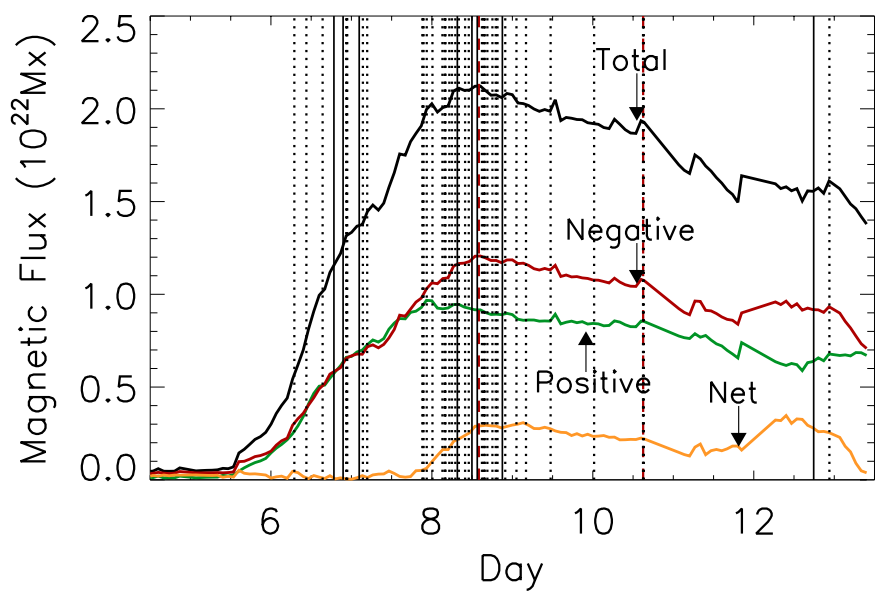

Fig. 3. Absolute values of the total unsigned (black), positive (green), negative (red) and net (yellow) magnetic fluxes of AR 11045. The vertical dotted lines represent the beginning times of the $35 \mathrm{C}$-class flares, and the vertical solid lines the beginning times of the $8 \mathrm{M}$-class flares. The red vertical dashed lines are the two times that separate the three different phases of the magnetic flux.

respectively. It is a classic "three-part" structure CME consisting of a bright front, a dark cavity and a bright compact core with span angles of $113.6^{\circ}$ and $102.3^{\circ}$ for STEREO A and B satellites, separately. Moreover, from the observations of LASCO C2 (see Fig. 4k) and C3 (see Fig. 41), we notice that the CME is a halo one with a span angle of $221^{\circ}$.

Employing the composites of simultaneous EUVI $195 \AA$ and COR1 white light running difference images displayed in Fig. 5, we studied the relationship of the solar activities on February 7 , 2010. The upper panels are the observations of STEREO-A, the lower panels the observations of STEREO-B. These show that the erupted filament is the bright compact core of the CME (see Figs. 5b-d). Moreover, a diffuse bright front ahead of the CME perfectly connects to the on-disk signature of the EUV wave (see Figs. $5 \mathrm{c}$ and d). It seems that the EUV wave is closely associated with the CME and its expanding flanks rather than the flare. The solar activities therefore consist of a flare, a filament eruption, a CME, and an EUV wave from inner corona to outer corona in space. The white line in Fig. 5a and the black line in Fig. 5e represent the eruption directions of the filament observed by STEREO A and B satellites, respectively. Along these lines, we measured the propagation distances of these activities, including the filament eruption, the CME and the EUV wave, from the source position of the flare. The different signs in Fig. 6 represent the propagation distances of the different solar activities, and the different colors the distances obtained from different telescopes. By using a linear fit to these observational values from 

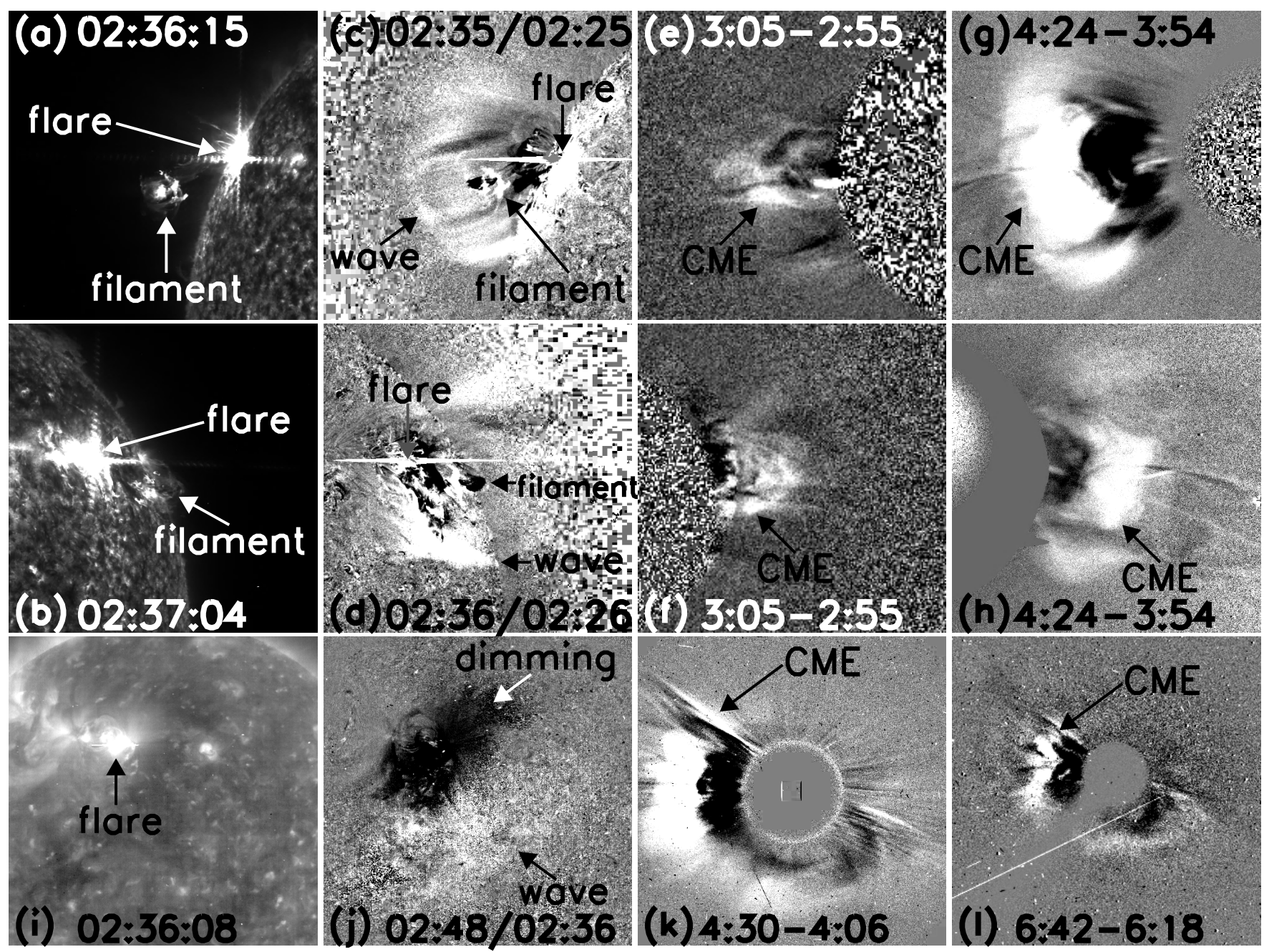

Fig. 4. STEREO/EUVI $304 \AA$ a) and b) and EIT $195 \AA$ i) images illustrating a flare and a filament eruption, EUVI $195 \AA$ c) and d) and EIT $195 \AA \mathbf{j}$ ) running ratio images, showing an EUV wave, and COR1 e) and f), COR2 g) and h), LASCO C2 k) and C3 l) running difference images, displaying a CME, on February 7, 2010. The upper panels are observations by the STEREO A satellite, the middle panels those by the STEREO B satellite, and the lower panels those by the SOHO satellite. The FOVs are $800^{\prime \prime} \times 800^{\prime \prime}$ in $\left.\mathbf{a}\right)-\mathbf{d}$ ), $3.1 R_{\odot} \times 3.1 R_{\odot}$ in e) and f), $7.7 R_{\odot} \times 7.7 R_{\odot}$ in $\mathbf{g}$ ) and $\left.\mathbf{h}\right), 1.4 R_{\odot} \times 1.4 R_{\odot}$ in i) and $\left.\mathbf{j}\right), 12.7 R_{\odot} \times 12.7 R_{\odot}$ in $\mathbf{k}$ ), and $35 R_{\odot} \times 35 R_{\odot}$ in $\mathbf{l}$ ), respectively.

STEREO A and B satellites, we obtained the average velocities of the filament eruption, the CME and the EUV wave, respectively. Under the assumption that the propagation distances are measured from the same point of the solar features from the observations of the STEREO A and B satellites, we obtained the real propagating velocities of the filament eruption, CME, and EUV wave of $284.8,555.4$ and $653.5 \mathrm{~km} \mathrm{~s}^{-1}$, separately. The solid curve in Fig. 6 shows the GOES soft X-ray flux. It indicates that the filament eruption and the EUV wave began near the peak time of the flare. Moreover, from the propagation distances and velocities of the filament eruption, CME and EUV wave, we also verified the spatial relationships among these activities displayed in Fig. 5.

It is well known that solar activities are always closely associated. Here, we statistically investigated the 29 filament eruptions and their associated activities, including flares, CMEs and EUV waves, and list the information in Table 2. Columns 13 in Table 2 show the dates, the initial times, and the velocities of the filament eruptions, Cols. 4-6 the initial times in STEREO/COR1, the velocities and the span angles of the associated CMEs, Cols. 7-8 the peak times and the classes (bigger than $C$ 1.0) of the associated flares, and Cols. 9-10 the initial times and the velocities of the associated EUV waves. It indicates that $23(79.3 \%)$ filament eruptions are associated with CMEs, $17(58.6 \%)$ are associated with bigger-than C 1.0 flares, and $10(34.5 \%)$ are associated with EUV waves.

\subsection{Jets in $A R 11045$}

Coronal jets are heated plasma ejections along open or largescale magnetic field lines. They are straight or slightly curved mass ejections stretching out and away from small flare-like brightenings at their footpoints. Employing the consecutive STEREO/EUVI $304 \AA$ running difference images in conjunction with the $304 \AA$ images, we chose the coronal jets which are the transient small-scale bright ejections that occurred in the AR to study in addition to the flares, CMEs, filament eruptions and EUV waves. Because of the different positions of these two STEREO satellites (see Fig. 1), we used the STEREO-B observations to explore the jets from February 5 to 8 , and the STEREO-A images for February 9 to 16. During the 12-day active phase of the AR, we detected 575 EUV jets with an occurrence rate of 49.3 per day, and found that lots of jets recurred. It is the first time that so many jets are verified in one AR during its active phase. Figure 7a displays an example of these 

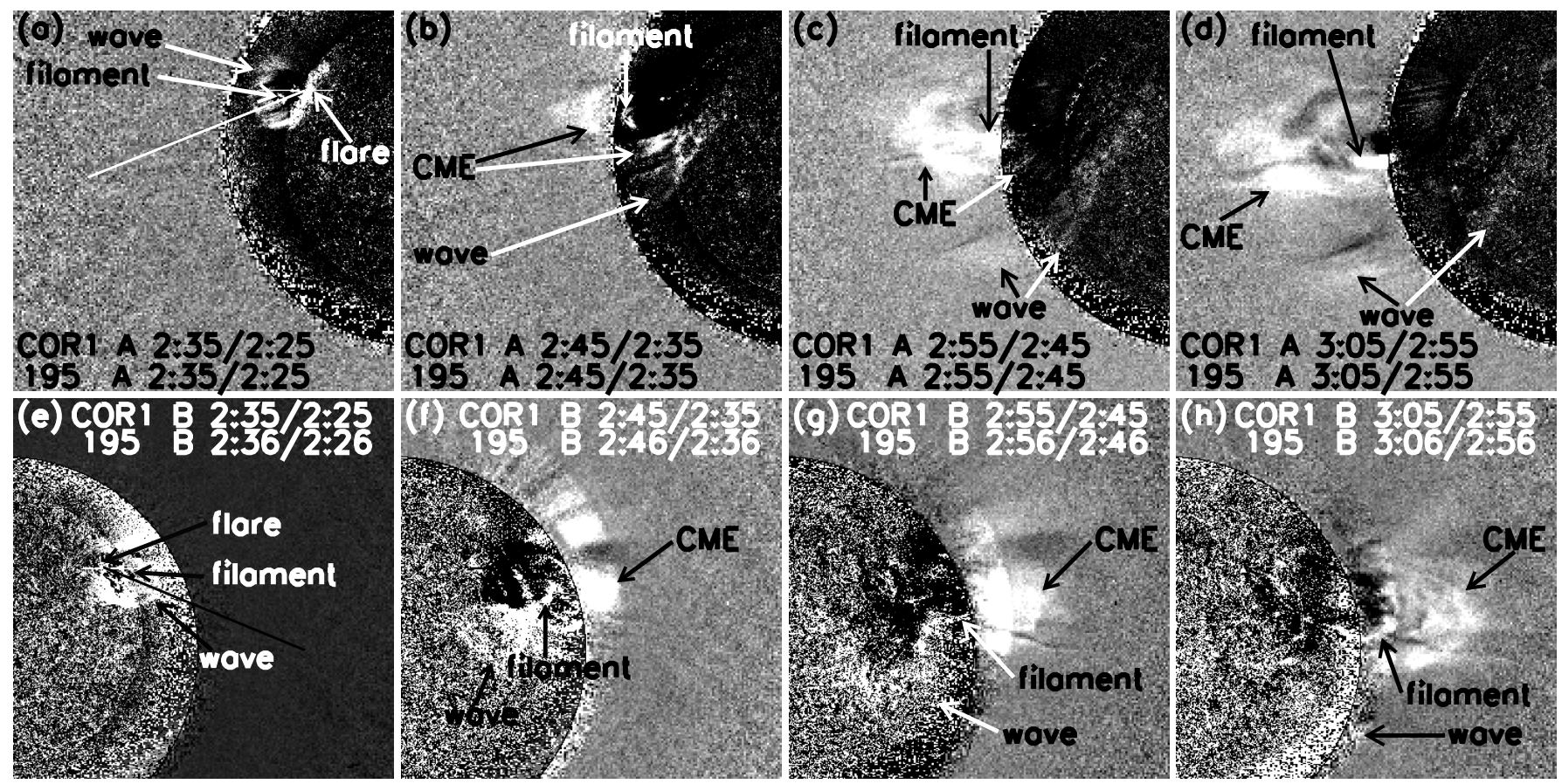

Fig. 5. Composites of EUVI $195 \AA$ and COR1 white light running difference images illustrating the evolutions of the flare, the filament eruption, the EUV wave and the CME. The upper panels are the STEREO-A observations, the lower panels the STEREO-B data. The white line in a) and the black line in e) present the eruption directions of the filament observed by STEREO A and B satellites, respectively. The FOV is $2.8 R_{\odot} \times 2.8 R_{\odot}$.

Table 1. Information on the M-class flares associated with the active region (AR) 11045.

\begin{tabular}{lcccccccc}
\hline \hline Date & Begin (UT) & Peak (UT) & End (UT) & Class & Position & CME & COR1 (UT) & Filament eruption \\
\hline $2010-02-06$ & $18: 47$ & $18: 59$ & $19: 09$ & M2.9 & N21E17 & Y & $19: 25$ & $\mathrm{~N}$ \\
$2010-02-06$ & $21: 31$ & $21: 37$ & $21: 42$ & M1.3 & N21E15 & Y & $22: 05$ & N \\
$2010-02-07$ & $2: 20$ & $2: 34$ & $2: 39$ & M6.4 & N21E10 & Y & $2: 45$ & Y \\
$2010-02-08$ & $7: 36$ & $7: 43$ & $7: 46$ & M4.0 & N23W05 & Y & $8: 05$ & N \\
$2010-02-08$ & $11: 57$ & $12: 03$ & $12: 06$ & M1.1 & N23W07 & N & $*$ & N \\
$2010-02-08$ & $13: 32$ & $13: 47$ & $13: 50$ & M2.0 & N23W08 & Y & $13: 45$ & N \\
$2010-02-08$ & $21: 01$ & $21: 23$ & $21: 28$ & M1.0 & N23W12 & N & $*$ & N \\
$2010-02-12$ & $17: 52$ & $18: 08$ & $18: 15$ & M1.1 & N28W53 & N & N & Y \\
\hline
\end{tabular}

Notes. The asterisk means there is no observation.

jets, indicated by “jet”, observed by STEREO-B/EUVI $304 \AA$ at 19:35 UT on February 7, 2010. At the root of the jet, there is a bright patch denoted by the red circle. Figure $7 \mathrm{~b}$ illustrates an EUVI-B $195 \AA$ Amage showing the same jet and the same bright patch at the root of the jet. Figure 7c shows a SOHO/MDI magnetogram at 19:15 UT, which is $20 \mathrm{~min}$ before the jet observation, and Fig. 7d the simultaneous SOHO/EIT $195 \AA$ image of the AR. To perform the co-alignments between STEREO-B and SOHO images, employing simultaneous $195 \AA$ images observed by EUVI-B and EIT (see Figs. 7b and d), we used recognition of solar features, such as bright patches, and marked them using the green numbers (see numbers 1-6 denoted in Figs. 7b and d). We coaligned the STEREO and SOHO $195 \AA$ images by eye. Then we coaligned the EUVI $304 \AA$ images with EUVI $195 \AA$ images, and MDI magnetograms with EIT $195 \AA$ images. The green numbers $1-6$ in Fig. 7 represent the respective bright patches. After the coalignments, we noticed that the "jet" indicated in Fig. $7 \mathrm{~d}$ denotes the same jet as shown in Figs. $7 \mathrm{a}$ and $\mathrm{b}$, the same was true for the red circle, which denotes the bright patch at the root of the jet. This shows that the jet is rooted at a negative magnetic field region near a positive one (see the red circle in Fig. 7c).
Using the same method as shown in Fig. 7, we coaligned the STEREO/EUVI and SOHO images to identify the roots of these EUV jets, and plot them on the SOHO/MDI magnetograms as pink pluses illustrated in Fig. 2. Figures $2 b-f$ display the distribution of the roots of these jets (see the pink pluses) in the AR from February 5 to 9 . In order to explore why there are so many jets, we researched the evolution of the magnetic fields at the roots of these jets from February 5 to 14 , and noticed that the jets were mostly rooted surrounding the emerging flux. Because there is no MDI observation of the AR after February 14, we cannot study the magnetic field evolution at the roots of the jets identified from February 15 to 16. However, from STEREO/EUVI observations, we note that the brightenings at the roots of these jets are to the west of the AR, where a positive magnetic field emerged west of the negative magnetic field F since February 8 (see Figs. 2e and f), which has already caused lots of jets since February 10 (see the peak of the number of the jets around 19:00 UT on February 10 displayed in Fig. 8a).

Furthermore, we overlayed the seven M-class flare cores from February 5 to 8 as colored contours on the magnetograms of the AR in Figs. 2c-e to investigate the spatial relationship between the jets and the flares, and notice that the jets occurred 


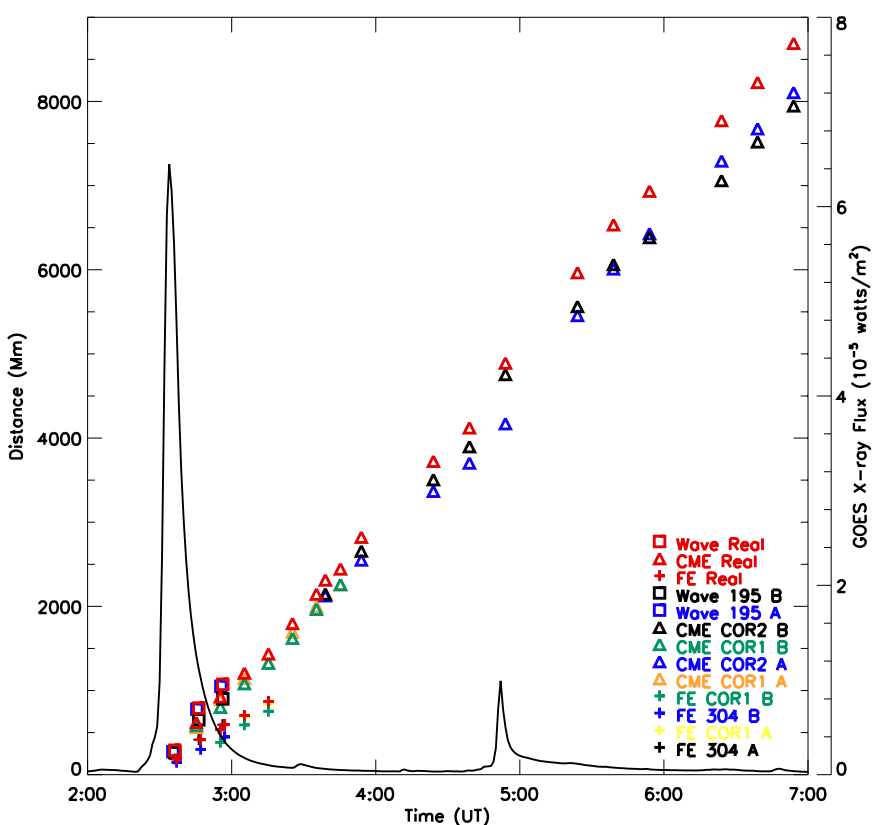

Fig. 6. Distance vs. time plots for the propagations of the filament eruption (FE, pluses), the CME (triangles), and the EUV wave (squares) for the event shown in Figs. 4 and 5. The solid curve presents the GOES soft X-ray flux.

mostly around these flare cores. In addition to the spatial relationship, we also studied the temporal relationship between the jets and the flares. During jet events, magnetic energy is released partly through radiation, partly through dynamical processes. Some jets may be associated with weak enhancements in soft X-ray emission, where the X-ray radiative energy is lower by 2 orders of magnitude compared with the kinetic energy. The jets therefore mainly display mass ejections, and the stored magnetic energy is mostly translated into the kinetic energy of the ejected material during the jet activities, rather than the thermal energy (Schmieder et al. 1988). Hence, the soft X-ray flux is mainly enhanced by the flares. The upper panel of Fig. 8 illustrates the number of these jets (the black lines) and the GOES-14 1-8 $\AA$ soft X-ray flux (the blue curve) during the evolution of the AR from 7:00 UT on February 5 to 0:00 UT on February 17. Here, the square signs represent the number of the jets in nine hours. It indicates that the jets occurred all the time along with the evolution of the AR. From February 5 to 13, because there was only AR 11045 active on the solar disk, the soft X-ray flux of the Sun almost represent that of AR 11045. During these nine days, we chose six intervals (see I-VI in Fig. 8) according to the maximum and the minimum phases of the soft X-ray flux, separated by the vertical dashed lines, to investigate the relationship between the number of the jets and the soft X-ray flux. It seems that the soft X-ray flux is inverse correlated with the number of the jets during these six intervals. That is to say, the higher the soft X-ray flux, the fewer the jets.

To examine the reliability that the soft X-ray flux is inverse correlated with the number of the jets, we chose other ARs to study under three criteria: 1 . the AR emerged during January 1 and March 30, 2010 around the time when AR 11045 existed on the solar disk, 2. there was no other AR when the chosen one existed on the solar disk, so the soft X-ray flux is contributed mainly by the chosen AR. 3. the AR nearly disappeared or became plages before it rotated out of the solar disk. Finally, we obtained AR 11045, which emerged on the solar disk

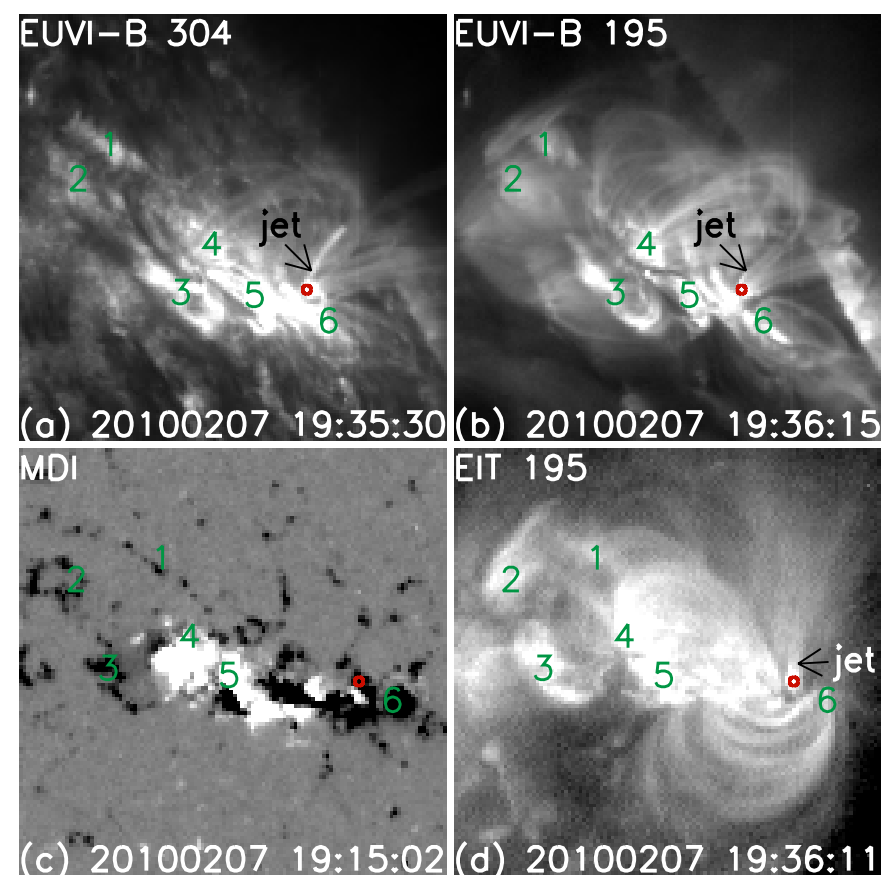

Fig. 7. STEREO-B/EUVI $304 \AA$ a), $195 \AA$ b) and SOHO/EIT $195 \AA$ d) images, showing a jet, and an MDI magnetogram c) of the AR. The red circles represent the bright patch at the root of the jet, and the green numbers 1-6 the same bright patches observed by STEREO-B and SOHO/EIT. The FOVs of $\mathbf{a}-\mathbf{b}$ ) and $\mathbf{c}-\mathbf{d}$ ) are $238.5^{\prime \prime} \times 238.5^{\prime \prime}$ and $315.6^{\prime \prime} \times 315.6^{\prime \prime}$, respectively.

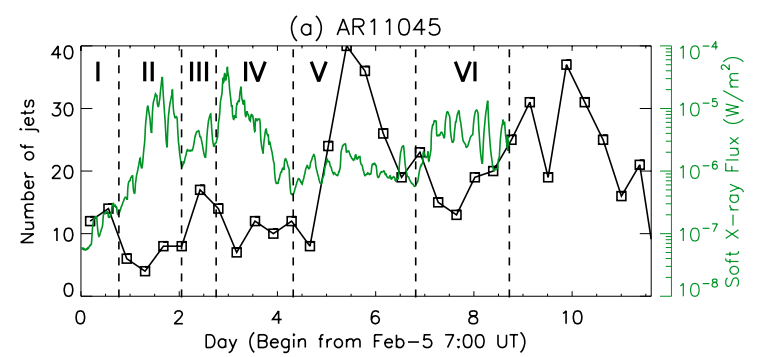

(b) AR11043

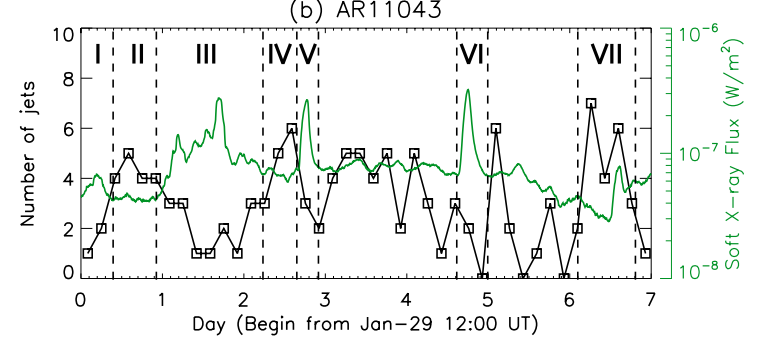

Fig. 8. a) Time series of the GOES-14 1-8 $\AA$ soft X-ray flux (the green curve) and the number (the square signs) of the jets in $9 \mathrm{~h}$ in AR 11045 . b) Same as a) but for the number (the square signs) of the jets in $4 \mathrm{~h}$ in AR 11045. The vertical dashed lines separate six regions I-VI in a) and seven regions (I-VII) in b) showing the time intervals we chose to study.

at heliographic N25 E50 from 17:39 UT on January 29, 2010, which we studied in comparison with AR 11045.

Figure 9 illustrates the formation and evolution of the magnetic fields of AR 11045. It shows that the AR is a simple bipole during its evolution, marked as $A$ and $B$ in Fig. 9. Similar to AR 11045, we studied the EUV jets that occurred in this AR using the STEREO-B/EUVI $304 \AA$ images from January 29 to 
Table 2. Information on the 29 filament eruptions and their associated solar activities in AR 11045.

\begin{tabular}{lccccccccc}
\hline \hline Date & $\mathrm{FE}(\mathrm{UT})$ & $V_{\mathrm{FE}}\left(\mathrm{km} \mathrm{s}^{-1}\right)$ & $\mathrm{CME}$ & $V_{\mathrm{CME}}$ & $\mathrm{SA}_{\mathrm{CME}}(\mathrm{Deg})$ & $F_{\mathrm{PT}}$ & $\mathrm{FC}$ & $E W$ & $V_{\mathrm{EW}}$ \\
\hline $2010-02-06$ & $1: 15$ & 99.1 & $1: 45$ & 400.1 & 16.1 & $\mathrm{~N}$ & $\mathrm{~N}$ & $\mathrm{~N}$ & $\mathrm{~N}$ \\
$2010-02-06$ & $2: 25$ & 80.6 & $3: 05$ & 297.6 & 91.1 & $\mathrm{~N}$ & $\mathrm{~N}$ & $\mathrm{~N}$ & $\mathrm{~N}$ \\
$2010-02-06$ & $5: 25$ & 42.3 & $\mathrm{~N}$ & $\mathrm{~N}$ & $\mathrm{~N}$ & $\mathrm{~N}$ & $\mathrm{~N}$ & $\mathrm{~N}$ & $\mathrm{~N}$ \\
$2010-02-06$ & $8: 06$ & 101.9 & $7: 25$ & 459.3 & 88.7 & $7: 04$ & $\mathrm{C} 4.0$ & $7: 05$ & 463.2 \\
$2010-02-06$ & $10: 56$ & 55.6 & $11: 05$ & 332.3 & 88.7 & $10: 48$ & $\mathrm{C} 1.0$ & $\mathrm{~N}$ & $\mathrm{~N}$ \\
$2010-02-06$ & $19: 56$ & 73.2 & $20: 05$ & 216.8 & 45.3 & $\mathrm{~N}$ & $\mathrm{~N}$ & $\mathrm{~N}$ & $\mathrm{~N}$ \\
$2010-02-07$ & $2: 36$ & 284.8 & $2: 45$ & 555.4 & 113.6 & $2: 34$ & $\mathrm{M} 6.4$ & $2: 36$ & 653 \\
$2010-02-07$ & $14: 56$ & 71.5 & $15: 45$ & 105.9 & 68.6 & $\mathrm{~N}$ & $\mathrm{~N}$ & $\mathrm{~N}$ & $\mathrm{~N}$ \\
$2010-02-07$ & $21: 36$ & 90.8 & $21: 45$ & 320.6 & 17.2 & $21: 39$ & $\mathrm{C} 4.2$ & $21: 25$ & 159.2 \\
$2010-02-07$ & $22: 35$ & 57.1 & $22: 45$ & 318.2 & 41.1 & $\mathrm{~N}$ & $\mathrm{~N}$ & $\mathrm{~N}$ & $\mathrm{~N}$ \\
$2010-02-08$ & $0: 26$ & 102.1 & $0: 45$ & 335.7 & 29.6 & $0: 16$ & $\mathrm{C} 1.4$ & $\mathrm{~N}$ & $\mathrm{~N}$ \\
$2010-02-08$ & $1: 16$ & 49.8 & $1: 45$ & 161.5 & 23.4 & $\mathrm{~N}$ & $\mathrm{~N}$ & $\mathrm{~N}$ & $\mathrm{~N}$ \\
$2010-02-08$ & $2: 36$ & 52.1 & $\mathrm{~N}$ & $\mathrm{~N}$ & $\mathrm{~N}$ & $\mathrm{~N}$ & $\mathrm{~N}$ & $\mathrm{~N}$ & $\mathrm{~N}$ \\
$2010-02-08$ & $3: 16$ & 219.4 & $3: 45$ & 310.7 & 83.5 & $3: 17$ & $\mathrm{C} 6.2$ & $3: 25$ & 222.1 \\
$2010-02-08$ & $3: 56$ & 75.4 & $4: 45$ & 326.1 & 83.3 & $3: 58$ & $\mathrm{C} 2.4$ & $4: 05$ & 232.8 \\
$2010-02-08$ & $4: 56$ & 81.8 & $5: 45$ & 203.2 & 47.4 & $5: 23$ & $\mathrm{C} 8.6$ & $5: 25$ & 221.7 \\
$2010-02-08$ & $5: 46$ & 56.5 & $6: 25$ & 205.2 & 32.9 & $6: 06$ & $\mathrm{C} 6.8$ & $\mathrm{~N}$ & $\mathrm{~N}$ \\
$2010-02-08$ & $11: 06$ & 116.7 & $11: 45$ & 165.2 & 31.4 & $11: 14$ & $\mathrm{C} 1.8$ & $\mathrm{~N}$ & $\mathrm{~N}$ \\
$2010-02-08$ & $13: 56$ & 144.2 & $13: 45$ & 326.1 & 11.8 & $13: 47$ & $\mathrm{M} 2.0$ & $\mathrm{~N}$ & $\mathrm{~N}$ \\
$2010-02-08$ & $15: 06$ & 86.5 & $15: 45$ & 310.1 & 22.6 & $15: 03$ & $\mathrm{C} 1.2$ & $15: 05$ & 372.2 \\
$2010-02-08$ & $16: 36$ & 89.4 & $\mathrm{~N}$ & $\mathrm{~N}$ & $\mathrm{~N}$ & $17: 07$ & $\mathrm{C} 1.3$ & $\mathrm{~N}$ & $\mathrm{~N}$ \\
$2010-02-08$ & $18: 16$ & 67.2 & $18: 45$ & 254.4 & 15.7 & $18: 08$ & $\mathrm{C} 1.3$ & $18: 35$ & 279.6 \\
$2010-02-09$ & $5: 06$ & 37.3 & $5: 45$ & 212.9 & 18.3 & $4: 17$ & $\mathrm{C} 2.4$ & $\mathrm{~N}$ & $\mathrm{~N}$ \\
$2010-02-09$ & $15: 26$ & 17.6 & $\mathrm{~N}$ & $\mathrm{~N}$ & $\mathrm{~N}$ & $\mathrm{~N}$ & $\mathrm{~N}$ & $\mathrm{~N}$ & $\mathrm{~N}$ \\
$2010-02-12$ & $17: 56$ & 65.2 & $\mathrm{~N}$ & $\mathrm{~N}$ & $\mathrm{~N}$ & $18: 08$ & $\mathrm{M} 1.1$ & $\mathrm{~N}$ & $\mathrm{~N}$ \\
$2010-02-12$ & $22: 06$ & 20.0 & $22: 30$ & 411.1 & 71.4 & $22: 40$ & $\mathrm{C} 3.0$ & $22: 55$ & 444.6 \\
$2010-02-13$ & $20: 46$ & 34.4 & $21: 25$ & 187.1 & 23.4 & $*$ & $*$ & $\mathrm{~N}$ & $\mathrm{~N}$ \\
$2010-02-14$ & $6: 36$ & 102.5 & $\mathrm{~N}$ & $\mathrm{~N}$ & $\mathrm{~N}$ & $*$ & $*$ & $\mathrm{~N}$ & $\mathrm{~N}$ \\
$2010-02-14$ & $21: 36$ & 32.3 & $22: 45$ & 206.1 & 27.1 & $*$ & $*$ & $\mathrm{~N}$ & $\mathrm{~N}$ \\
\hline
\end{tabular}

Notes. The "FE" and " $V_{\mathrm{FE}}$ " illustrate the filament eruption and the mean velocity of filament eruption, and " $V_{\mathrm{CME}}$ " and "SA $\mathrm{C}_{\mathrm{CME}}$ " the mean velocity

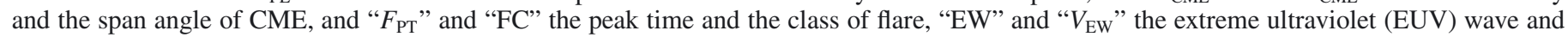
the mean velocity of EUV wave. "N" means no activity is detected, and the asterisk no observations.

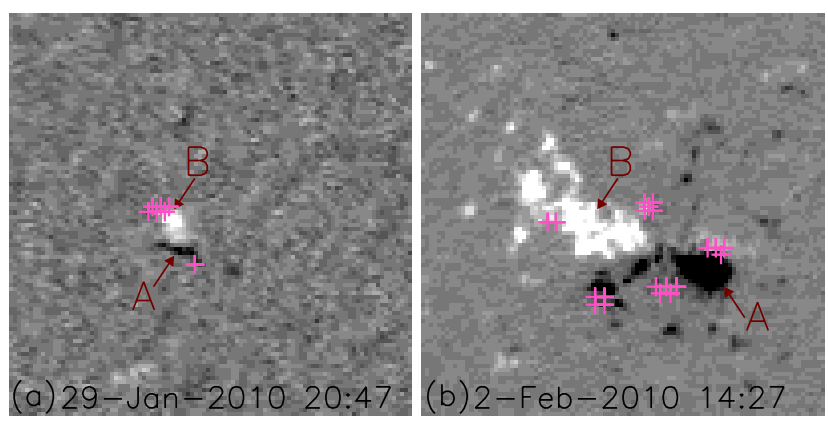

Fig. 9. Time series of MDI magnetograms showing the formation and evolution of the magnetic fields of AR 11045. A-B mark the emerged magnetic fields. The pink pluses show the roots of the jets on the magnetograms. The FOV is $200^{\prime \prime} \times 200^{\prime \prime}$.

February 2, and the STEREO-A/EUVI $304 \AA$ observations from February 3 to 14 . Finally, 373 jets were detected during 17 days, with a daily occurrence rate of 22 , which is about half that of AR 11045. Using both the SOHO and STEREO/EUVI observations, we identified the roots of these jets, and plot them on the SOHO/MDI magnetograms as pink pluses in Figs. 9a and $b$, which illustrate the distributions of the roots of the jets on January 29 and February 2, 2010, respectively. These show that the jets occurred surrounding the AR. We investigated the evolution of the magnetic fields at the roots of the jets, and also found that the jets are mostly rooted around the emerging flux of AR 11045, consistent with the result obtained for AR 11045.
Because only several B-class flares occurred in this AR, it is difficult for us to identify the flare positions using 10 min cadence observations. However, as we all know, the flare usually occurs in the AR near the sunspots along the neutral lines. Therefore, the jets may also have occurred around the flare cores, similar to the spatial relationship between the flares and the jets derived from AR 11045. From January 29 to February 5, 2010, because only one AR 11045 existed on the solar disk, the soft X-ray flux of the Sun mainly represents that of the AR. During the seven days, we studied the temporal relationship between the number of the jets and the soft X-ray flux, and show them in the lower panel of Fig. 8. We chose seven intervals (see I-VII in Fig. 8b) according to the maximum and minimum phases of the soft X-ray flux, separated by the vertical dashed lines, and found that the soft X-ray flux is also inverse correlated with the number of the jets during these seven intervals, identical to the temporal relationship between the flares and the jets obtained from AR 11045.

Along with the AR evolution, we investigated the solar activities, such as flares, CMEs, filament eruptions and jets that are associated with the evolution of the magnetic flux, and list the results in Table 3. Column 1 shows the solar activities, Cols. 2-4, $5-7$, and $8-10$ the number, the percentage, and the daily rate of the activities during the emergence phase, the stable phase, and the decaying phase of the magnetic flux, respectively, and Cols. 11-12 the number and the daily rate of the solar activities during the active phase of the AR. Here, the end time of the decaying phase is 0:00 UT on February 15 for flares, when there were no soft X-ray data of the AR, and 0:00 UT on February 17 
Table 3. The solar activities associated with the three different phases of the magnetic flux of AR 11045 .

\begin{tabular}{|c|c|c|c|c|c|c|c|c|c|c|c|}
\hline \multirow[b]{2}{*}{ Activities } & \multicolumn{3}{|c|}{ Emergence phase } & \multicolumn{3}{|c|}{ Stable phase } & \multicolumn{3}{|c|}{ Decaying phase } & \multicolumn{2}{|r|}{ Total } \\
\hline & Num. & Percentage & DR & Num. & Percentage & DR & Num. & Percentage & DR & Num. & DR \\
\hline Flares & 26 & $60.5 \%$ & 8.0 & 14 & $32.6 \%$ & 6.9 & 3 & $6.9 \%$ & 0.7 & 43 & 4.4 \\
\hline CMEs & 24 & $45.3 \%$ & 7.4 & 6 & $11.3 \%$ & 2.9 & 23 & $43.4 \%$ & 3.6 & 53 & 4.5 \\
\hline Filament Eruptions & 19 & $65.5 \%$ & 5.8 & 5 & $17.25 \%$ & 2.4 & 5 & $17.25 \%$ & 0.8 & 29 & 2.5 \\
\hline Jets & 88 & $15.3 \%$ & 27.1 & 76 & $13.2 \%$ & 37.2 & 411 & $71.5 \%$ & 64.5 & 575 & 49.3 \\
\hline
\end{tabular}

Notes. "Num." means the number of activities, and "DR" the daily rate of activities.

for other activities. From the table, we notice that $93.1 \%$ flares (see also the dotted (the C-class flares) and solid (the M-class flares) lines in Fig. 3) and 82.75\% filament eruptions occurred in the emergence and stable phases of the magnetic flux. However, $43.4 \%$ CMEs and $71.5 \%$ jets occurred in the decaying phase of the magnetic flux, even though there were less flares. Moreover, excluding the jets, the occurrence rates of the solar activities per day are higher in the emergence phase of the magnetic flux. In addition, the daily rate of the jets is also inverse correlated with that of the flares.

\section{Conclusions and discussion}

We have studied the formation and evolution of the magnetic fields of the first productive AR 11045 of SC 24, and its associated solar activities, and found that during the evolution of the AR, there are six pairs of main emerging magnetic fields, in addition to some small-scale magnetic field emergences. Furthermore, we discovered significant magnetic cancelations and shear motions that occurred among these emerging magnetic fields. Associated with the magnetic field evolution are $35 \mathrm{C}$ class and $8 \mathrm{M}$-class flares, $53 \mathrm{CMEs}, 29$ filament eruptions and 19 EUV waves. Moreover, most of the flares (93.1\%) and filament eruptions $(82.75 \%)$ occurred in the emergence and stable phases of the AR. However, $43.4 \%$ CMEs and $71.5 \%$ jets occurred in the decaying phase, when there were less flares $(6.9 \%)$ and filament eruptions (17.25\%). In an example of eight M-class flares, the activities consisted of a flare, a filament eruption, a CME and an EUV wave from inner corona to outer corona in space, and the filament eruption and EUV wave occurred near the peak time of the flare. We statistically investigated the relationship between the 29 filament eruptions and their associated activities, and found that $79.3 \%$ filament eruptions are associated with CMEs, $58.6 \%$ associated with flares, and $34.5 \%$ associated with EUV waves. In addition to these solar activities, we identified 575 EUV jets in this AR, with a daily occurrence rate of 49.3. It is the first time that so many jets have been observed in one AR during its active phase. We statistically studied these jets along with the magnetic field evolution, and found that the jets mostly occurred surrounding the emerging flux of the AR. Additionally, we detected magnetic field dynamics, such as emergence, cancelation, and shear motion at the roots of these jets. We also investigated the spatial and temporal relationships between the jets and the flares, and noticed that the jets mostly occurred around the flare cores, and the soft X-ray flux is inverse correlated with the number of the jets, especially in the beginning nine days since the appearance of the AR. In comparison to AR 11045, we studied another newly emerging AR 11043, and detected 373 EUV jets during 17 days, with an occurrence rate of 22 ones per day, about half that in AR 11045. We investigated these 373 jets along with the magnetic field evolution, as well as the relationships between the jets and the flares, and obtained similar results to those derived for AR 11045.

Obviously the flares are prolific and violent during the AR growth, and diminish considerably during the decaying phase. However, CMEs continued at a high level during the decaying phase, similar to the result of Demoulin et al. (2002), who found that the rate of CMEs per day was constant, even if there was no flare in the decaying phase. This may indicate that the number of the flares depends more on the free energy that is available locally, while the CME production depends more on global quantities, in particular on the amount of magnetic helicity (Low 1997; Demoulin et al. 2002).

The relationship between filament eruptions and other phenomena of solar activities has already been investigated (Subramanian \& Dere 2001; Hori \& Culhane 2002; Zhou et al. 2003). Gilbert et al. (2000) found that $94 \%$ of the eruptive filaments had an associated CME, and Hori \& Culhane (2002) proposed that $92 \%$ of the prominence eruptions were associated with CMEs. Gopalswamy et al. (2003) reported that $83 \%$ of the radial events had a strong correlation to the CMES. However, Jing et al. (2004) concluded that $56 \%$ of the filament eruptions were associated with CMEs. We found that $79.3 \%$ of the filament eruptions are associated with CMEs, consistent with Gopalswamy et al. (2003) (83\%), lower than Gilbert et al. (2000) (94\%) and Hori \& Culhane (2002) (92\%), but higher than Jing et al. (2004) (56\%). Furthermore, Jing et al. (2004) reported that AR filament eruptions had a flare association of $95 \%$, which is higher than ours $(58.6 \%)$. This may be caused by the sample of the flares we used because we only chose flares bigger than C 1.0 to investigate the relationship between filament eruptions and flares. We investigated the spatial and temporal relationships among the flare, filament eruption, CME, and EUV wave. Apparently the EUV wave is closely associated with the CME rather than the flare, and our findings may support the conclusion of Veronig et al. (2010) that a dome-shaped large-scale coronal EUV wave is consistent with the spherical expansion and decay of a weakly shocked fast-mode MHD wave.

Even though there are many studies about jets (e.g. Brueckner \& Bartoe 1983; Schmahl 1981; Zhang et al. 2000; Liu \& Kurokawa 2004), for the first time we studied the jets that occurred in one AR during its evolution, which represent the lower magnetic reconnection processes occurring between the emerged magnetic fluxes and preexisting ambient fields. We found a daily occurrence rate (49.3 jets per day) of the jets in AR 11045 higher than twice that (22 jets per day) of AR 11045. Comparing the magnetic field evolutions of ARs 11045 and 11043, we found that the significant differences between the daily occurrence rates of the jets in these two ARs may be caused by two reasons. One is that the AR 11045 is more complex, with multiple-polar magnetic fields, than AR 11045, which is a simple bipole. The other one is that there are more new magnetic field emergences in AR 11045 than in the AR 11045. 
Schmieder et al. (1993) suggested that surges do not originate from precisely the same part of an AR as the flares. Jiang et al. (2007) reported that the homologous jets were associated with small flares at the same base, but their exact footpoints were spatially separated from the flare. Through investigating the jets in two ARs, we found that the jets occurred mostly around the emerging flux of the ARs. Furthermore, the jets are rooted at different places from the flares, usually around the flare cores. This seems to be natural because flares and jets (or surges) are signatures of energy release in structures of different nature (Schmieder et al. 1988). Jets are observed when the energy is released in an open structure, whereas flares occur if a significant amount of energy goes into magnetically-closed structures. Recently, ubiquitous hot plasma outflows at the edges or periphery of ARs have been discovered (Sakao et al. 2007; Baker et al. 2009; Tian et al. 2011). It seems that the huge number of jets in this study are spatially consistent with the outflows. This may support the results of the three-dimensional numerical simulations of Harra et al. (2011), who found that the reconnection jets are a mechanism that drives the outflows that appear at the edges of the ARs. Moreover, the number of the jets is inverse correlated with the soft X-ray flux, which is mainly enhanced by the flares. This inverse correlation may be caused by the transformation of magnetic energy into either kinetic (for jets) or thermal energy (for flares), because the flares are the signatures of magnetic reconnection, allowing the temperature to rise (Wang et al. 2002; Qiu \& Gary 2003), while in the jet events, there is a transformation of magnetic energy into kinetic energy (Schmieder et al. 1995). In addition, the distribution of the jets along with the magnetic field evolution, and the spatio-temporal relationships between the jets and the flares may well represent a scenario of two-step reconnection, proposed first by Wang \& Shi (1993) for flare observations, and confirmed by Chae et al. (1999) for EUV explosive events. Because the new emergence is usually lower, the first-step reconnection takes place in the photosphere, or lower atmosphere, when the new magnetic field emerges. It is slow but continuous since the conductivity in the photosphere is much lower than that of the fully-ionized plasma in the upper chromosphere and corona. This slow reconnection manifests itself as flux cancelation observed in the photospheric magnetograms (Livi et al. 1985; Martin et al. 1985), and the magnetic reconnection in the lower atmosphere may convert the magnetic energy into heat and kinetic energy, displayed as jets or surges; while, at the same time, it can transport the magnetic energy and complexity into the rather large-scale magnetic structure higher in the corona, e.g. along the overlying magnetic field lines involved in the jet magnetic reconnection processes. Of course, other magnetic dynamics, such as shearing motions and rotations of the sunspots, will also accumulate magnetic energy in the higher magnetic structures in the corona (Li \& Zhang 2009). The second-step reconnection can only take place when some critical status is achieved in the large-scale magnetic structure higher in the corona. This reconnection is explosive in nature, which is directly responsible for the energy released in the transient solar activities, which appear as flares (Zhang et al. 2000). In this study, the jets were rooted at the region with magnetic field emergence and cancelation, consistent with the results of Kurokawa \& Kawai (1993), Schmieder et al. (1995), Chae et al. (1999), Zhang et al. (2000) and Liu \& Kurokawa (2004). All these observations support the magnetic reconnection jet (surge) model (Roy 1973a; Shibata et al. 1992; Canfield et al. 1996) that the magnetic reconnection between a newly emerging flux and a preexisting ambient magnetic field is the essential mechanism of jet production. Shibata et al. (1992) and Yokoyama \& Shibata $(1995,1996)$ demonstrated in their two-dimensional MHD numerical simulations that magnetic reconnection between emerging magnetic fluxes and preexisting fields indeed produce adjacent hot and cold plasma ejections simultaneously.

Most of the large-scale magnetic fluxes are believed to emerge from the solar interior in the form of $\Omega$ loops (Magara \& Longcope 2001; Fan 2001), such as the magnetic fields $E$ and $F$, $G$ and $H, I$ and $J$, and $K$ and $L$, illustrated in Fig. 2. In addition, most of the magnetic field dynamics are associated with EUV brightenings or solar activities. However, from the SOHO/EIT and STEREO/EUVI observations we were unable to identify any brightening or activity associated with the cancelation between the emerged opposite polarity magnetic fields $B$ and $C$ (see also Fig. 2b), which suggests that this cancelation is caused by the rising of a U-shaped loop (van Dreil-Gesztelyi et al. 2000; Pariat et al. 2009b). For this reason, the magnetic fields $A-D$ are two adjacent $\Omega$ loops connected below the photosphere by a U-shaped loop.

Based on the observations, we displayed schematic diagrams in Fig. 10 to explain the formation and evolution of the AR. Figures 10a-d illustrate the magnetic field structures at four different times corresponding to Fig. 2. In these figures, the parallelograms represent the surface of the photosphere, and the solid lines the magnetic flux tubes above the photosphere; the dotted lines the magnetic flux tubes under the photosphere; the gray circles the positive magnetic fields; the black circles the negative magnetic fields. Before the emergence of the AR, there were five main magnetic flux tubes below the surface of the photosphere, among which the black one was strongly toroidal with two adjacent $\Omega$ structures (see Fig. 10a). From the middle of February 5, 2010, the first $\Omega$ structure of the black flux tube rose from below the photosphere. Then the second $\Omega$ structure also emerged into the photosphere. As a result, the first and the second pairs of magnetic fields $A$ and $B$, and $C$ and $D$ appeared, as shown in Fig. 10a. The emerging two adjacent $\Omega$ loops lifted the U-loop, connecting these two $\Omega$ loops in the solar interior, above the photosphere. Therefore, magnetic fields $B$ and $C$ were thrown together, which is shown as "clean" cancelation (van Dreil-Gesztelyi et al. 2000), and disappeared at last. Following the emergences of the black magnetic flux tube, the second (the red curve) and the third (the green curve) magnetic flux tubes emerged into the photosphere (see Fig. 10b). Figures 10c and d demonstrate the emergence of the fourth (the blue curve) and fifth (the pink curve) magnetic flux tubes, respectively. Along with the rise of these flux tubes, significant shear motions and cancelations between the positive and negative magnetic fields occurred. Finally, the AR became a relatively simple bipole, as shown in Fig. 10d. However, Pariat et al. (2004) proposed that coronal loops do not result from the smooth emergence of large-scale $\Omega$ loops below the photosphere, but rather from the rise of undulatory flux tubes whose upper parts emerge because of the Parker instability and whose dipped lower parts emerge because of magnetic reconnection (Magara 2001; Pariat et al. 2007). As the successive emergence of bipoles in AR 11045 show, the rise of undulatory flux tubes may be another alternative model for the formation of the AR. The complicated evolution of the first productive AR 11045 of SC 24, including magnetic field emergences, cancelations and shear motions, is the reason why there are so many flares, CMEs, filament eruption, EUV waves and abundant jets (Wang \& Shi 1993; Zhang et al. 2001b).

Because the time cadence of STEREO/EUVI $304 \AA$ observations is $10 \mathrm{~min}$, those EUV jets, whose lifetimes are less than 

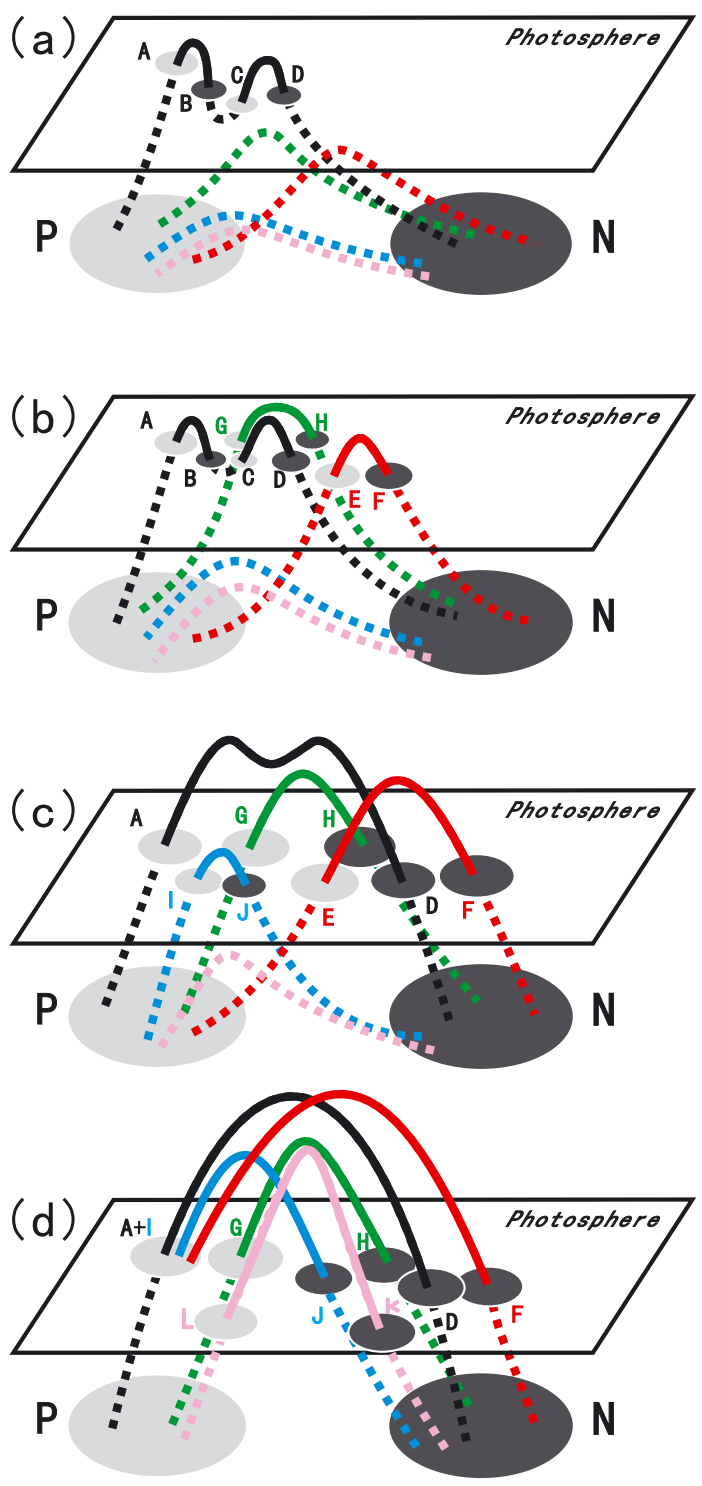

Fig. 10. Schematic diagrams illustrating the formation and evolution of the magnetic field structures of AR 11045. The parallelograms represent the surface of the photosphere, and the gray (black) circles the positive (negative) magnetic fields, the solid (dotted) lines the magnetic flux tube above (under) the photosphere. A-L mark the emerged magnetic fields, and $\mathrm{P}$ and $\mathrm{N}$ the footpoints of these magnetic flux tubes.

$10 \mathrm{~min}$, will not be identified. With more higher spatio-temporal resolution observations of ARs and their associated solar activities, we will study the AR evolutions and the relationship between the AR evolution and its associated activities in more detail. Additional investigation about the spatial and the temporal relationships between the jets and the flares is also planned.

Acknowledgements. The authors are indebted to the STEREO, SOHO and GOES teams for providing the data. The work is supported by the National Natural Science Foundations of China (G11003026, 40890161, 11025315, 41074123, 10873020, 10973019, and 40974112), and the National Basic Research Program of China under grant G2011CB811403, and Young Researcher Grant of National Astronomical Observatory, Chinese Academy of Sciences.

\section{References}

Alexander, D., \& Fletcher, L. 1999, Sol. Phys., 190, 167 Archontis, V., Tsinganos, K., \& Gontikakis, C. 2010, A\&A, 512, L2
Aschwanden, M. J., Fletcher, L., Schrijver, C. J., \& Alexander, D. 1999, ApJ, 520,880

Baker, D., van Driel-Gesztelyi, L., Mandrini, C. H., et al. 2009, ApJ, 705, 926

Bhatt, N. J., Jain, R., \& Aggarwal, M. 2009, Sol. Phys., 260, 225

Brueckner, G. E., \& Bartoe, J.-D. F. 1983, ApJ, 272, 329

Brueckner, G. E., Howard, R. A., Koomen, M. J., et al. 1995, Sol. Phys., 162, 357

Canfield, R. C., Peardon, K. P., Leka, K. D., et al. 1996, ApJ, 464, 1016

Chae, J., Qiu, J., Wang, H., et al. 1999, ApJ, 513, L75

Chifor, C., Young, P. R., Isobe, H., et al. 2008, A\&A, 481, L57

Conlon, P. A., James McAteer, R. T., Gallagher, P. T., \& Fennell, L. 2010, ApJ, 722,577

Cook, J. W., Rutten, R. J., \& Hoekzema, N. M. 1996, ApJ, 470, 647

Delaboudinière, J. P., Artzner, G. E., Brunaud, J., et al. 1995, Sol. Phys., 162, 291

Démoulin, P., Mandrini, C. H., van Drial-Gesztelyi, L., et al. 2002, A\&A, 382, 650

Fan, Y. 2001, ApJ, 554, L111

Gibson, S. E., Fletcher, L., Del Zanna, G., et al. 2002, ApJ, 574, 1021

Gilbert, H. R., Holzer, T. E., Burkepile, J. T., \& Hundhausen, A. J. 2000, ApJ, 537, 503

Golub, L., Rosner, R., Vaiana, G. S., \& Weiss, N. O. 1981, ApJ, 243, 309

Gopalswamy, N., Shimojo, M., Lu, W., et al. 2003, ApJ, 586, 562

Harra, L. K., Archontis, V., Pedram, E., et al. 2011, Sol. Phys., 352

Harrison, R. A. 1986, A\&A, 304, 585

Hori, K., \& Culhane, J. L. 2002, A\&A, 382, 666

Howard, R. A., Moses, J. D., Vourlidas, A., et al. 2008, Space Sci. Rev., 136, 67

Isobe, H., Yokoyama, T., Shimojo, M., et al. 2002, ApJ, 566, 528

Jiang, J., \& Wang, J. X. 2007, MNRAS, 377, 711

Jiang, Y. C., Chen, H. D., Li, K. J., Shen, Y. D., \& Yang, L. H. 2007, A\&A, 469, 331

Jibben, P., \& Canfield, R. C. 2004, ApJ, 610, 1129

Jing, J., Yurchyshyn, V. B., Yang, G., et al. 2004, ApJ, 614, 1054

Kumar, P., Srivastava, A. K., Filippov, B., Erdélyi, R., \& Uddin, W. 2011, Sol. Phys., 272, 301

Kurokawa, H., \& Kawai, G. 1993, in The Magnetic and Velocity Fields of Solar Active Region, ed. H. Zirin, G. Ai, \& H. Wang, ASP Conf. Ser., 46, 507

Li, K. J. 2009, Res. Astron. Astrophys., 9, 959

Li, L. P., \& Zhang, J. 2009, ApJ, 706, L17

Lites, B. W., Skumanich, A., \& Martinez, P. V. 1998, A\&A, 333, 1053

Liu, Y., \& Kurokawa, H. 2004, ApJ, 610, 1136

Liu, R., \& Wang, H. M. 2009, ApJ, 703, L23

Livi, S. H. B., Wang, J., \& Martin, S. F. 1985, Aust. J. Phys., 38, 855

Low, B. C. 1997, in Coronal mass ejections, Geophysical Monograph 99, ed. N. Crooker, J. A. Joselyn, \& J. Feynman, 39

Magara, T. 2001, ApJ, 549, 608

Magara, T., \& Longcope, D. W. 2001, ApJ, 559, L55

Martin, S. F., \& Harvey, K. H. 1979, Sol. Phys., 64, 93

Martin, S. F., Livi, S. H. B., \& Wang, J. 1985, Aust. J. Phys., 38, 929

Miesch, M. S. 2005, Liv. Rev. Sol. Phys., 2

Nakariakov, V. M., \& Ofman, L. 2001, A\&A, 372, L53

Newton, H. W. 1934, MNRAS, 94, 472

Pariat, E., Aulanier, G., Schmieder, B., et al. 2004, ApJ, 614, 1099

Pariat, E., Schmieder, B., Berlicki, A., et al. 2007, A\&A, 473, 279

Pariat, E., Antiochos, S. K., \& DeVore, C. R. 2009a, ApJ, 691, 61

Pariat, E., Masson, S., \& Aulanier, G. 2009b, ApJ, 701, 1911

Patterson, A., \& Zirin, H. 1981, ApJ, 243, L99

Qiu, J., \& Gary, D. E. 2003, ApJ, 599, 615

Roy, J.-R. 1973a, Sol. Phys., 28, 95

Roy, J.-R. 1973b, Sol. Phys., 32, 139

Rust, D. M. 1968, in Structure and Development of Solar Active Regions, ed. K. O. Kiepenheuer, IAU Symp., 35, 77

Rust, D. M., Webb, D. F., \& MacCombie, W. 1977, Sol. Phys., 54, 53

Sakao, T., Kano, R., Narukage, N., et al. 2007, Science, 318, 1585

Scherrer, P. H., Bogart, R. S., Bush, R. I., et al. 1995, Sol. Phys., 162, 169

Schmahl, E. J. 1981, Sol. Phys., 69, 135

Schmieder, B., Mein, P., Martres, M. J., \& Tandberg-Hanssen, E. 1984, Sol. Phys., 94, 133

Schmieder, B., Simnett, G. M., Tangberg-Hanssen, E., \& Mein, P. 1988, A\&A, 201, 327

Schmieder, B., van Driel-Gesztelyi, L., Gerler, O., et al. 1993, Sol. Phys., 146, 163

Schmieder, B., Golub, L., \& Antiochos, S. K. 1994, ApJ, 425, 326

Schmieder, B., Shibata, K., van Driel-Gesztelyi, L., et al. 1995, Sol. Phys., 156, 245

Schmieder, B., Heinzel, P., Wiik, J. E., Lemen, J., \& Hiei, E. 1996, Adv. Spac. Res., 17, 111

Schrijver, C. J. 2009, Adv. Spac. Res., 43, 5, 739 
Sheeley, N. R. Walters, J. H., Wang, Y. M., \& Howard, R. A. 1999, J. Geophys. Res., 104, 24739

Shibata, K., Ishido, Y., Acton, L. A., et al. 1992, PASJ, 44, L173

Shimojo, M., Hashimoto, S., Shibata, K., et al. 1996, PASJ, 48, 123

Shimojo, M., Shibata, K., \& Harvey, K. L. 1998, Sol. Phys., 178, 379

Sterling, A. C., \& Moore, R. L. 2005, ApJ, 630, 1148

Subramanian, P., \& Dere, K. P. 2001, ApJ, 561, 372

Tandberg-Hanssen, E. 1977, in Illustrated Glossary for Solar and Solar Terrestrial Physics (Dordrecht, Holland: D. Reidel Publ. Co.), ed. A. Bruzek, \& C. J. Durrant, 106

Thomas, R. J., Crannell, C. J., \& Starr, R. 1995, Sol. Phys., 95, 323

Tian, H., McIntosh, S. W., \& De Pontieu, B. 2011, ApJ, 727, L37

Tripathi, D., Isobe, H., \& Mason, H. E. 2006, A\&A, 453, 1111

Tsuneta, S., Acton, L. W., Bruner, M., et al. 1991, Sol. Phys., 136, 37

van Dreil-Gesztelyi, L., Malherbe, J. M., \& Démoulin, P. 2000, A\&A, 364, 845

Veronig, A. M., Muhr, N., Kienreich, I. W., et al. 2010, ApJ, 716, L57
Wang, Y., \& Sheeley, Jr. N. R. 2002, ApJ, 575, 542

Wang, J. X., \& Shi, Z. X. 1993, Sol. Phys., 143, 119

Wang, H. M., Goode, P. R., Denker, C., et al. 2000, ApJ, 536, 971

Wang, H. M., Spirock, T. J., Qiu, J., et al. 2002, ApJ, 576, 497

Wang, T. J., Solanki, S. K., Innes, D. E., Curdt, W., \& Marsch, E. 2003, A\&A, 402, L17

Webb, D. F., Cliver, E. W., Crooker, N. U., Cry, O. C. St., \& Thompson, B. J. 2000, J. Geophy. Res., 105, 7491

Yokoyama, T., \& Shibata, K. 1995, Nature, 375, 42

Yokoyama, T., \& Shibata, K. 1996, PASJ, 48, 353

Zhang, J., Wang, J. X., \& Liu, Y. 2000, A\&A, 361, 759

Zhang, J., Dere, K. P., Howard, R. A., Kundu, M. R., \& White, S. M. 2001a, ApJ, 559, 452

Zhang, J., Wang, J. X., Deng, Y. Y., \& Wu, D. J. 2001b, ApJ, 548, L99

Zhang, J., Wang, J. X., \& Nitta, N. 2001c, Chin. J. Astron. Astrophys., 1, 85

Zhou, G. P., Wang, J. X., \& Cao, W. D. 2003, A\&A, 397, 1057 